\title{
Combined Petrosal Approach for Resection of Petroclival Meningioma with Endoscopic Assistance: Operative Video and Technical Nuances
}

\author{
James K. Liu ${ }^{1}$ Vincent N. Dodson ${ }^{1}$ \\ ${ }^{1}$ Department of Neurological Surgery, Center for Cerebrovascular \\ and Skull Base Surgery, Rutgers University, New Jersey Medical \\ School, Neurological Institute of New Jersey, RWJ Barnabas Health, \\ Livingston and Newark, New Jersey, United States
}

\begin{abstract}
Address for correspondence Dr. James K. Liu, MD, Department of Neurological Surgery, Rutgers University, New Jersey Medical School, 90 Bergen Street, Suite 8100, Newark, NJ 07103, United States (e-mail: james.liu.md@rutgers.edu).
\end{abstract}

J Neurol Surg B 2019;80(suppl S3):S292-S293.

\begin{abstract}
Petroclival meningiomas are formidable lesions due to their deep location in the skull base and proximity to critical neurovascular structures. The combined petrosal approach, comprised of an anterior petrosectomy and posterior retrolabyrinthine petrosectomy, allows for both supra- and infratentorial exposure of the tumor in the petroclival region while potentially preserving hearing function (-Fig. 1). In this operative video atlas manuscript, the authors demonstrate a step-by-step technique for microsurgical resection of a petroclival meningioma via the combined petrosal approach. The nuances of microsurgical and skull base technique are illustrated including drilling of the petrous bone, transecting the tentorium, resection of the tumor, and multilayered reconstruction of the dural defect. The combined petrosal

Keywords

- combined petrosal

- meningioma

- petroclival

- endoscopic approach allows for multiple angles of attack including both subtemporal and presigmoid corridors. In summary, the combined petrosal approach with endoscopic assistance is an important strategy in the armamentarium for surgical resection of petroclival meningiomas (-Fig. 2).

The link to the video can be found at: https://youtu.be/ipZb9kHcP2g.
\end{abstract}

Conflict of Interest

The authors have no conflicts of interest to declare.

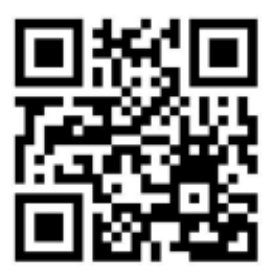

received

March 25, 2019

accepted after revision

March 25, 2019

published online

April 30, 2019 www.thieme.com/skullbasevideos

www.thieme.com/jnlsbvideos
License terms

10.1055/s-0039-1688487. ISSN 2193-6331. (c) 2019 Georg Thieme Verlag KG Stuttgart · New York

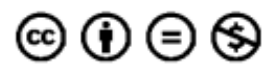




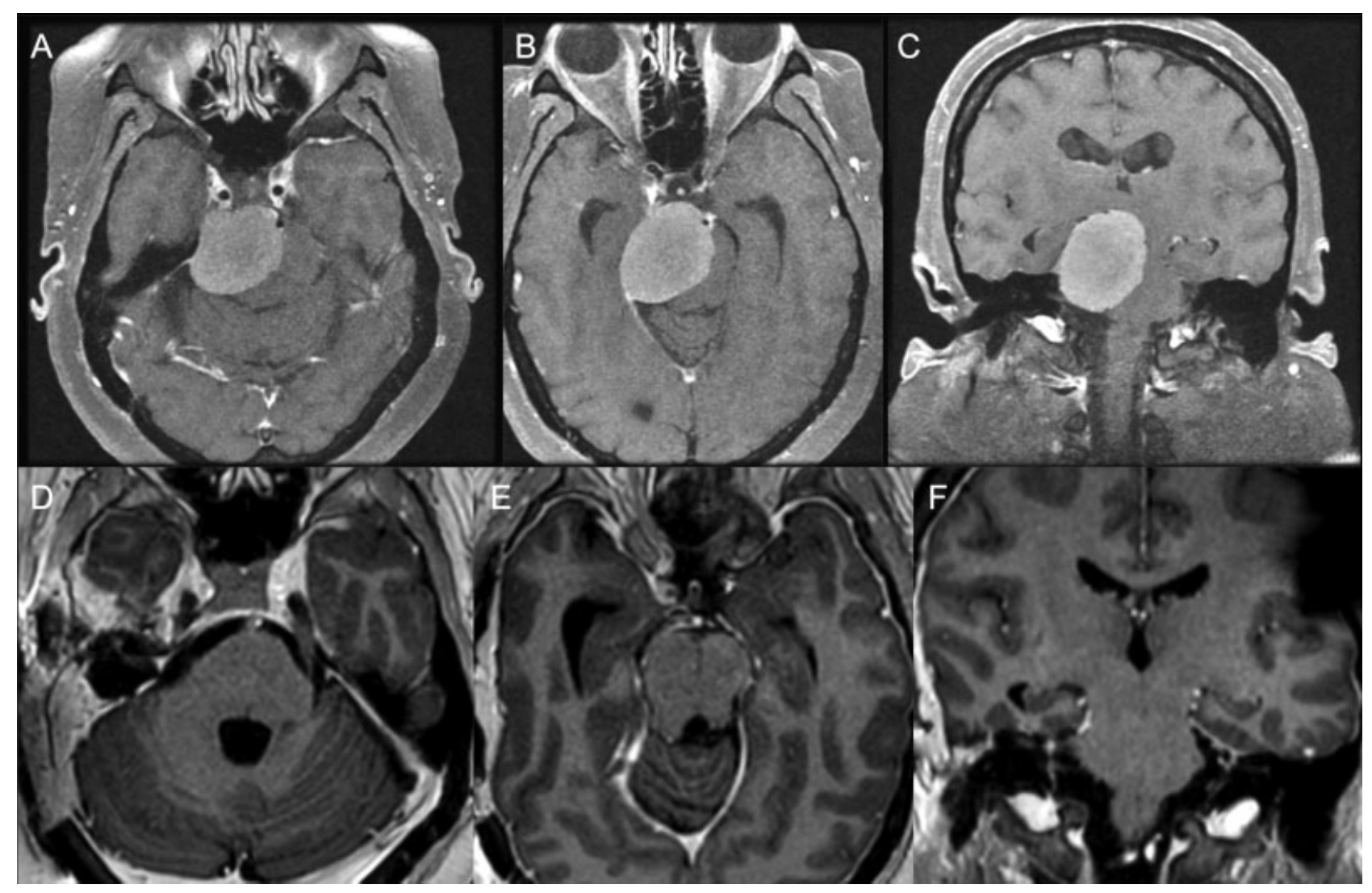

Fig. 1 (A, B) Preoperative post-gadolinium T1-weighted axial (A and B) and coronal (C) MRI demonstrates a right petroclival meningioma with severe brainstem compression and hydrocephalus. (D, E) 3-month postoperative post-gadolinium T1-weighted axial (D and E) and coronal (F) MRI demonstrates successful gross total resection of the tumor with decompression and re-expansion of the brainstem. The hydrocephalus was resolved with a ventriculoperitoneal shunt. MRI, magnetic resonance imaging.

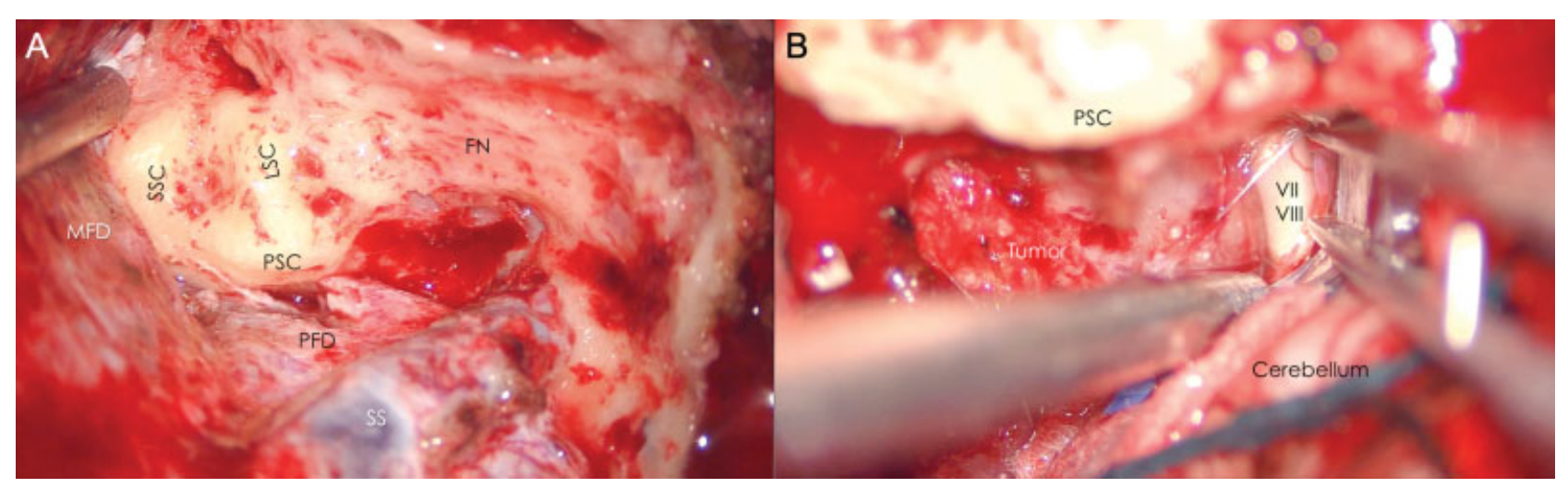

Fig. 2 (A) Intraoperative photograph of a right combined petrosal approach displaying important anatomical landmarks. From left to right, top to bottom: middle fossa dura (MFD), superior semicircular canal (SSC), lateral semicircular canal (LSC), facial nerve (FN), posterior semicircular canal (PSC), posterior fossa dura (PFD), sigmoid sinus (SS). (B) View of the tumor situated cephalad to cranial nerves VII and VIII via the combined petrosal exposure. 